Niebelschütz, Florentina; Cimalla, Volker; Brückner, Klemens; Stephan, Ralf; Tonisch, Katja; Hein, Matthias A.; Ambacher, Oliver:

\title{
Sensing applications of micro- and nanoelectromechanical resonators
}

URN: $\quad$ urn:nbn:de:gbv:ilm1-2014210301

Published OpenAccess: November 2014

Original published in:

Proceedings of the Institution of Mechanical Engineers / N. - London : Sage Publ (ISSN 2041-3092). - 221 (2007) 2, S. 59-65.

DOI: $\quad 10.1243 / 17403499 J N N 100$

URL: $\quad$ http://dx.doi.org/10.1243/17403499JNN100

[Visited: 2014-10-14]

„Im Rahmen der hochschulweiten Open-Access-Strategie für die Zweitveröffentlichung identifiziert durch die Universitätsbibliothek IImenau."

"Within the academic Open Access Strategy identified for deposition by IImenau University Library."

„Dieser Beitrag ist mit Zustimmung des Rechteinhabers aufgrund einer (DFG-geförderten) Allianz- bzw. Nationallizenz frei zugänglich."

"This publication is with permission of the rights owner freely accessible due to an Alliance licence and a national licence (funded by the DFG, German Research Foundation) respectively."

\section{DFG}

Nationallizenzen 


\title{
Proceedings of the Institution of Mechanical Engineers, Part N: Journal of Nanoengineering and Nanosystems \\ http://pin.sagepub.com/
}

\section{Sensing applications of micro- and nanoelectromechanical resonators}

F Niebelschütz, V Cimalla, K Brückner, R Stephan, K Tonisch, M A Hein and O Ambacher Proceedings of the Institution of Mechanical Engineers, Part N: Journal of Nanoengineering and Nanosystems 2007221 : 59

DOI: $10.1243 / 17403499 J N N 100$

The online version of this article can be found at:

http://pin.sagepub.com/content/221/2/59

\author{
Published by: \\ @SAGE \\ http://www.sagepublications.com
}

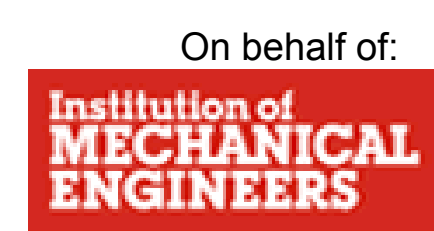

Institution of Mechanical Engineers

Additional services and information for Proceedings of the Institution of Mechanical Engineers, Part N: Journal of Nanoengineering and Nanosystems can be found at:

Email Alerts: http://pin.sagepub.com/cgi/alerts

Subscriptions: http://pin.sagepub.com/subscriptions

Reprints: http://www.sagepub.com/journalsReprints.nav

Permissions: http://www.sagepub.com/journalsPermissions.nav

Citations: http://pin.sagepub.com/content/221/2/59.refs.html

>> Version of Record - Jun 1, 2007

What is This? 


\title{
Sensing applications of micro- and nanoelectromechanical resonators
}

F Niebelschütz, V Cimalla, K Brückner, R Stephan, K Tonisch, M A Hein, and 0 Ambacher

Center for Micro- and Nanotechnologies (ZMN), Technische Universität Ilmenau, Ilmenau, Germany

The manuscript was received on 23 February 2007 and was accepted after revision for publication on 26 September 2007.

DOI: $10.1243 / 17403499 J N N 100$

\begin{abstract}
The sensitivity of micro- and nanoscale resonator beams for sensing applications in ambient conditions was investigated. Micro-electromechanical (MEMS) and nanoelectromechanical systems (NEMS) were realized using silicon carbide (SiC) and polycrystalline aluminium nitride (AlN) as active layers on silicon substrates. Resonant frequencies and quality factors in vacuum as well as in air were measured. The sensitivity behaviour under ambient conditions with a mass loading in the range of picogram (pg) was verified and measurements with biological mass loading were performed. In addition, the sensitivity to pressure variations was analysed.
\end{abstract}

Keywords: micro-electromechanical systems (MEMS), nanoelectromechanical systems (NEMS), biological sensing, resonator

\section{INTRODUCTION}

In the last few years, MEMS have become an important mainstream technology with a wide range of applications [1, 2]. The applications of these devices include microactuators, radio frequency (RF) MEMS, and micro-optoelectromechanical systems (MOEMS), as well as chemical $[\mathbf{3}]$, biological $[\mathbf{4}, \mathbf{5}]$, gas $[\mathbf{6}-\mathbf{9}]$, and microfluidic sensors [10].

As a result of the fact that small active masses enhance the sensitivity for very small mass loadings, a trend of miniaturization into the submicron domain has also set in.

Since increasing attention is drawn to the employment of materials, tools, and technologies for the fabrication of miniature sensors and actuators and their integration with electronic circuits to produce smart devices and systems, new materials such as wide-band-gap semiconductors have found their way into MEMS technology. One of the main advantages of wide-band-gap semiconductors is the very high mechanical, thermal, chemical, and biochemical

*Corresponding author: Zentrum für Mikro- und Nanotechnologien, Technische Universität Ilmenau, Gustav-Kirchhoff-Str. 7, Ilmenau, D-98693, Germany.email: florentina.niebelschuetz@ tu-ilmenau.de stability, which enables sensing applications in ranges that are not accessible with silicon (Si) technology.

In this work, the authors demonstrate MEMS and NEMS resonators, which are driven by a magnetomotive actuation under ambient conditions. This paper shows first, results proving the high sensitivity of the resonators under ambient conditions. The sensing properties for pressure variations have been verified. In addition, mass loadings using protein solution and cell cultures are described.

\section{FABRICATION OF RESONATORS}

Resonators based on AlN and SiC active layers were using the technology described in references [11] and [12]. SiC layers of $200 \mathrm{~nm}$ thickness were grown heteroepitaxially on Si (100) substrates using highvacuum chemical vapour deposition (HVCVD) at relatively low temperatures of about $900{ }^{\circ} \mathrm{C}$ and a base pressure of $1 \times 10^{-9}$ mbar [13]. The internal inhomogeneous and homogeneous strains, which directly influence the quality factors and resonant frequencies, mainly depend on the chosen growth temperature and so provide an opportunity of strain control in the active layer.

AlN layers of different thicknesses (150-300 nm) were deposited by reactive sputtering on Si (100) 


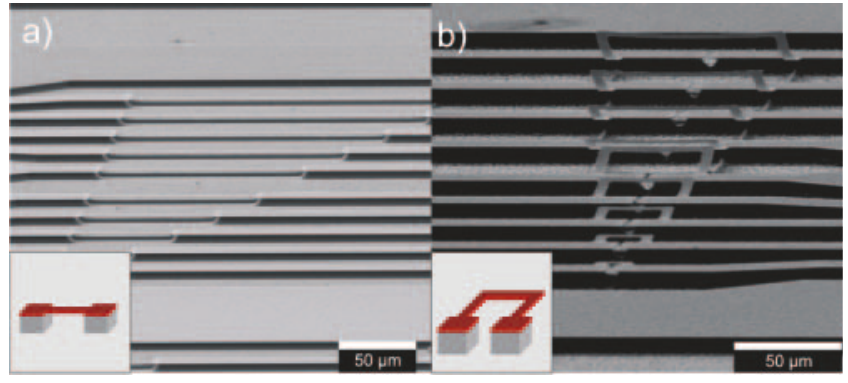

Fig. 1 Processed (a) doubly clamped and (b) coupled singly clamped AlN beams. The insets sketch the layout of the beams

substrates. The growth temperature was varied from 100 to $500^{\circ} \mathrm{C}$ resulting in a variation of the internal strain from $5 \times 10^{-6}\left(100^{\circ} \mathrm{C}\right)$ to $5 \times 10^{-5}\left(500^{\circ} \mathrm{C}\right)$.

Optical lithography was used to define freestanding singly and doubly clamped resonators with beam lengths of 10 to $500 \mu \mathrm{m}$ and widths of 0.9 to $8 \mu \mathrm{m}$, respectively (Figs. 1(a)) and 1(b)). Further decreasing of the beam width down to about $100 \mathrm{~nm}$ was achieved by e-beam lithography. The top electrode necessary for magnetomotive actuation consisted either of an evaporated titanium/gold $(\mathrm{Ti} / \mathrm{Au})$ or a chromium/gold $(\mathrm{Cr} / \mathrm{Au})$ layer system $(10 \mathrm{~nm} / 50 \mathrm{~nm}$, respectively), patterned via lift-off.

$\mathrm{The} \mathrm{Ti}(\mathrm{Cr}) / \mathrm{Au}$ layer served as the conductive layer as well as etch mask for the fluorine-based dry etching process in the case of $\mathrm{SiC}[\mathbf{1 4}, \mathbf{1 5}]$. The chlorine-based dry etching of the AlN layers required an additional $\mathrm{Ni}$ layer $(70 \mathrm{~nm})$ as etching mask. An inductively coupled plasma reactive ion etching (ICP RIE) system was used with a gaseous combination of $\mathrm{Ar}$ and $\mathrm{Cl}_{2}$ at a base pressure of $8 \times 10^{-3} \mathrm{mbar}$ and a substrate temperature of $20^{\circ} \mathrm{C}$ [16]. The anisotropic etching of $\mathrm{SiC}$ in a sulphur hexafluoride $\left(\mathrm{SF}_{6}\right)$, $\mathrm{Ar}$, and $\mathrm{O}_{2}$ mixture, as well as the isotropic etching of the silicon substrate in a $\mathrm{SF}_{6}$ and Ar mixture, were performed in an electron cyclotron resonance reactive ion etching (ECR RIE) system.

\section{MEASUREMENT SET-UP}

The resonators were operated by magnetomotive actuation. The mechanical oscillation of the beams is caused by an oscillating Lorentz force, which is generated from an applied alternating current along the beam placed within a permanent magnetic field. Since the induced response of the resonators is smaller than the exciting signal by four to seven orders of magnitude, detection is quite challenging. Therefore, a measurement technique was employed that separates the response from the excitation in the time domain [17]. The resonator was excited by short voltage pulses, and the amplified response

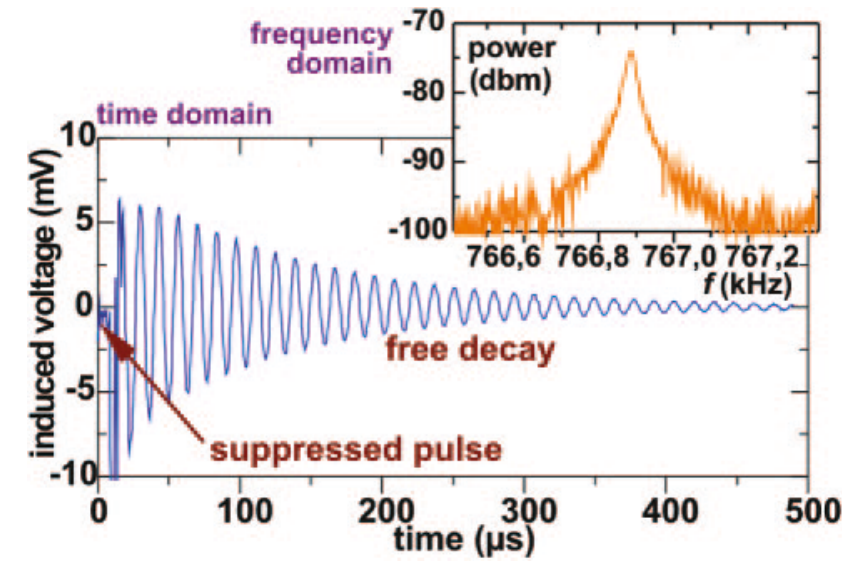

Fig. 2 Amplitude decay of a 200- $\mu$ m long AlN resonator versus time. Inset: measurement of the response of the same resonator in the frequency domain

signal was monitored by a digital oscilloscope. This method allows observation of the free decay of the oscillations, from which the resonant frequency and the quality factor can be determined according to

$$
Q=\frac{\pi \cdot f_{\mathrm{res}}\left(\tau_{2}-\tau_{1}\right)}{\ln U_{1} / U_{2}}
$$

where $T$ is the period of oscillation, and $f_{\text {res }}=1 / T$. $U_{1}$ and $U_{2}$ are the amplitudes of the envelope at times $\tau_{1}$ and $\tau_{2}$, respectively.

In addition, a balanced or bridge measurement technique, similar to the one described in reference [18], has been successfully developed for characterization in the frequency domain, too [19]. Here, a sine sweep over a certain frequency domain is generated and simultaneously applied to the resonant sensor and a reference resistor of the same value as the ohmic impedance of the resonator beam. Smallest differences of both impedances which ideally only occur near the resonant frequency of the beam can be visualized on a spectrum analyzer after differential amplification. The quality factor, $Q$, can then be calculated by $[\mathbf{2 0}]$

$$
Q=\frac{f_{\text {res }}}{b}
$$

where $b$ is the frequency bandwidth at $-3 \mathrm{~dB}$. Resonant frequencies and quality factors measured in both the time and frequency domains have been found to agree very accurately [19].

The time domain response of a typical beam resonator is displayed in Fig. 2, and, for the same resonator, the frequency response is also shown in the inset.

\section{SENSING APPLICATIONS}

The sensing properties of the resonator beams were investigated in detail, both for the detection of small 


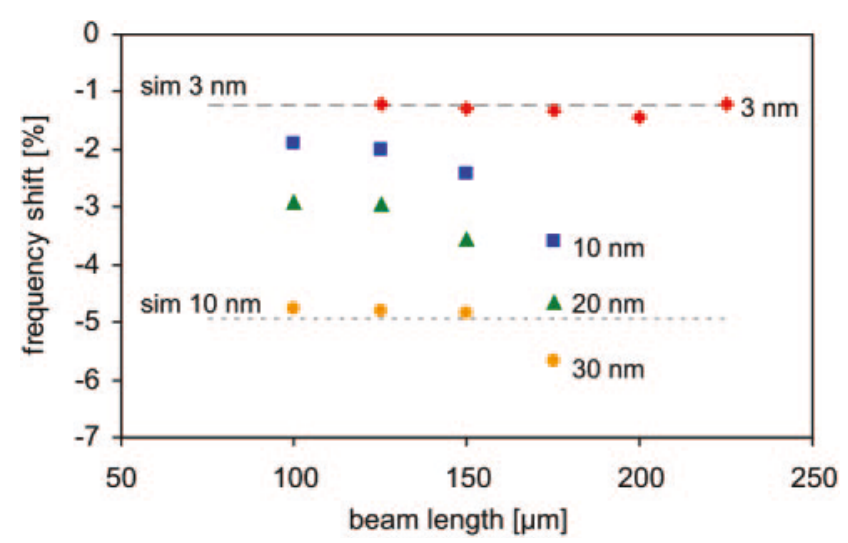

Fig. 3 Relative frequency shift after mass loading with a 3-nm Pd layer on SiC beams (red diamonds) and 10(blue squares), 20-(green triangles), and 30-nm thick (yellow dots) metal layers on AlN beams. The dashed and dotted lines show the calculated frequency shift for 3-nm and 10-nm thick metal layers, respectively

masses of a few pg with the feasibility for biological sensing, and for the determination of physical parameters such as pressure.

The resonators were characterized at ambient conditions and under vacuum. The measured resonant frequencies of the $\mathrm{AlN}$ and $\mathrm{SiC}$ beams were in the range of $50 \mathrm{kHz}$ to $7 \mathrm{MHz}$ and 0.32 to $2 \mathrm{MHz}$, respectively. The values of the resonant frequencies were naturally influenced by film thickness, beam length, and internal homogeneous strain of the active layer. The quality factor, $Q$, of the processed $\mathrm{AlN}$ and $\mathrm{SiC}$ beams was found to be as high as 350 to 500 in air and 500050000 in vacuum pressure $\left(p<5 \times 10^{-5}\right)$, respectively.

\section{MASS LOADING}

The response of the resonator beam on mass loading follows a linear dependence described by basic equation

$$
\Delta f_{\text {res }}=-\frac{1}{2} f_{\text {res }} \frac{\Delta m}{m}
$$

The change of the resonant frequency $f_{\text {res }}$ is determined by the ratio of the mass load $\Delta m$ to the mass $m$ of the beam. For a systematic study of the sensitivity, a homogeneous mass loading was achieved by sputtering thin layers of 3 to $4 \mathrm{~nm}$ palladium (Pd) on doubly clamped $\mathrm{SiC}$ resonators, corresponding to a mass loading of several picograms. The thickness of the Pd film can be controlled with an accuracy of better than $1 \mathrm{~nm}$. Figure 3 (red diamonds) shows a constant relative frequency shift of about -1.25 per cent, which illustrates the expected linear response of the beam on homogeneous mass loading. The mass loading determined by the

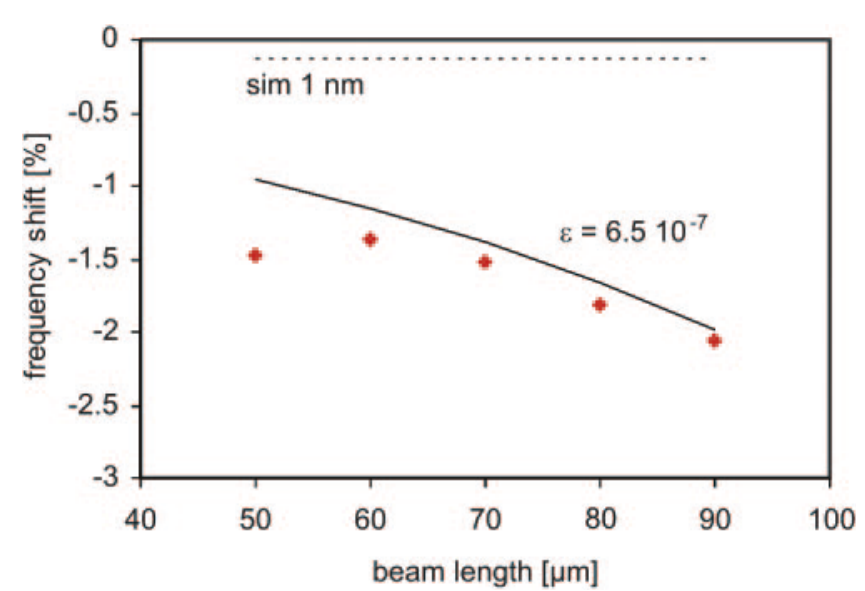

Fig. 4 Relative frequency shift after protein adhesion (red diamonds). The dotted line represents the calculated frequency shift for mass loading, and the solid line is the calculated frequency shift influenced by additional compressive strain

frequency shift is in excellent agreement with the calculated frequency shift (Fig. 3, dashed line).

However, when the mass was increased from $20 \mathrm{pg}$ up to $250 \mathrm{pg}$, by sputtering thin Pd layers of 10 to $30 \mathrm{~nm}$ in thickness on doubly clamped AlN resonators, strong deviations from the linear dependence were observed, due to the formation of a continuous metal layer on top of the resonator beam. Above a thickness of about $5 \mathrm{~nm}$, the sputtered metal forms a continuous, functional layer, which adds stress to the layer system and alters additionally the frequency. Consequently, equation (3) cannot be used for sensing of mass loadings in form of continuous layers on the resonators. Thus, the doubly clamped beams are suitable for sensing of monolayers, with very good agreement to simulated results, while additional strain caused by a continuous layer strongly influences the frequency behaviour and also the frequency shift, which could not be modelled, yet.

A series of mass loading experiments with molecular monolayers was performed using a protein monolayer of bovine serum albumin (BSA) with a planar density of $450 \mathrm{ng} / \mathrm{cm}^{2}$. Doubly clamped AlN resonator beams were exposed to the protein solvent phosphate buffered saline (PBS). The AlN-resonators with a width and thicknesses of $4 \mu \mathrm{m}$ and $230 \mathrm{~nm}$, respectively, have shown an original natural frequency of 0.5 to $1.3 \mathrm{MHz}$. After rinsing, a protein monolayer on the resonator beams is left, while the proteins preferably adhere at the Au surface of the conductive layer on top of the beams.

Figure 4 shows the resulting frequency shift after adhesion of the protein monolayer, due to the additional stress caused by a closed protein monolayer (red diamonds). The frequency shift calculated by means of equation (3), displayed as a dotted line, shows 


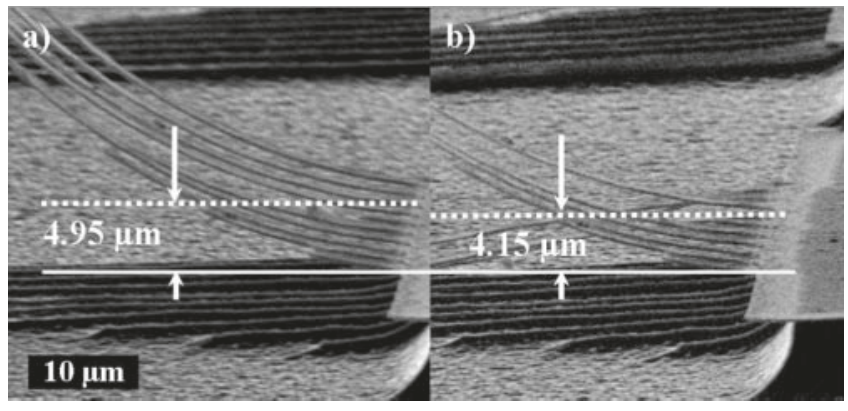

Fig. 5 SEM pictures of AlN cantilevers (a) without and (b) with protein adhesion showing clearly measurable strain effects

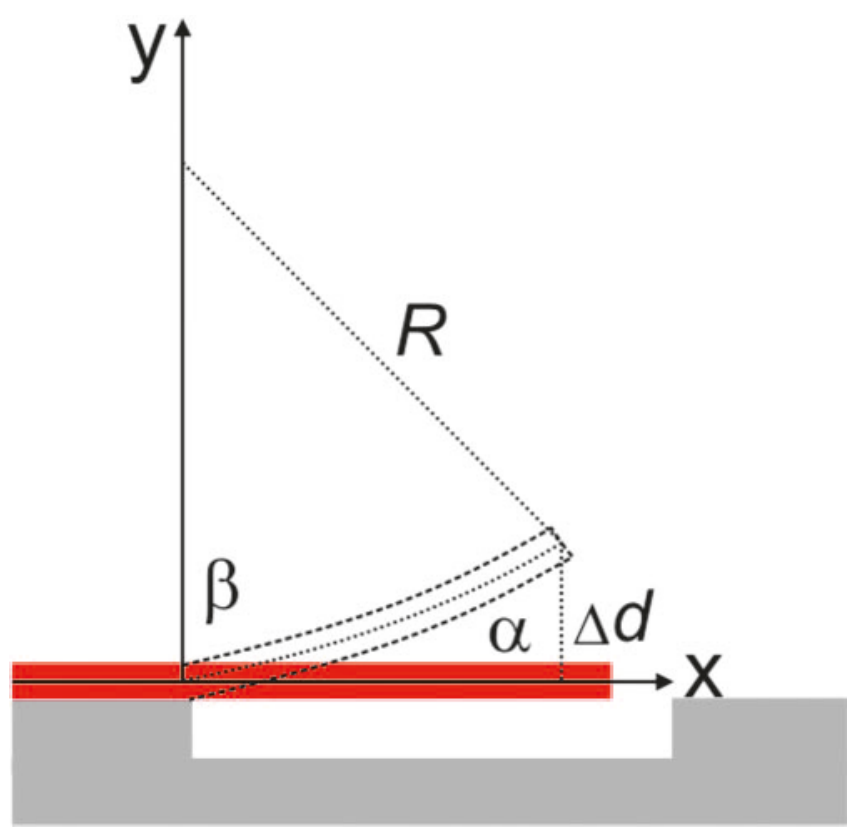

Fig. 6 Scheme of parameters used for the calculations of the internal strain

an obvious deviation from the measured results. Since the shape of the curve resembles the curves of the strain-influenced frequency shifts caused by homogeneous mass loading of continuous metal layers, further investigations were done concerning the internal strain. To evaluate the additional strain, which is brought into the system by the protein monolayer, simultaneously prepared singly clamped cantilevers were studied in terms of a detectable change of inhomogeneous strain.

Figure 5 shows SEM pictures of AlN cantilevers with and without protein adhesion. The height differs by about 15 per cent, which is a proof of altered strain within the system.

The stress $\sigma$ is related to the bending of the cantilever by

$$
\sigma=E \frac{d_{S}}{6 R d} \quad \text { and } \quad \sigma=\varepsilon E
$$

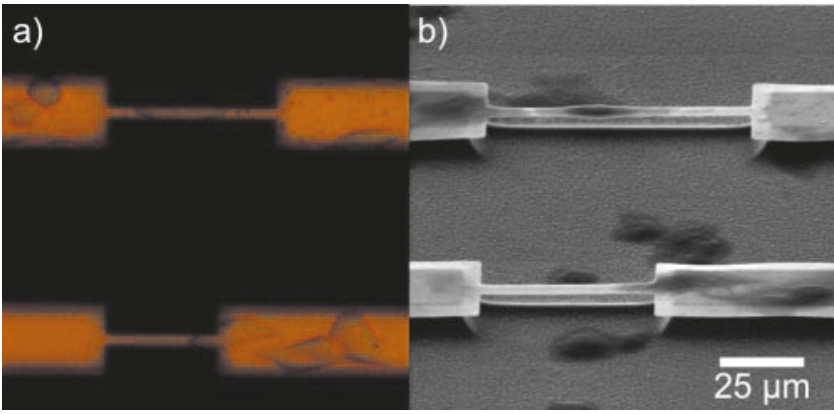

Fig. 7 (a) Optical microscope picture and (b) SEM picture of AlN beams loaded by $\mathrm{CHO}$ cells

which allows converting the height difference $\Delta d$ into the additional tension $\Delta \sigma$, where $d_{s}$ and $d$ are the thicknesses of the substrate and the protein layer, respectively. $R$ is the radius of curvature that can be calculated from

$$
R=\frac{L}{2 \cos \beta} \quad \text { and } \quad \beta=90^{\circ}-\arctan \frac{\Delta d}{L}
$$

The key parameters used in this derivation are displayed in Fig. 6. The additional internal strain was estimated to be $\varepsilon=6.5 \times 10^{-7}$. The frequency shift caused by the additional compressive strain is represented by the solid line in Fig. 4, according to [20]

$$
f_{\mathrm{res}}=1028 \sqrt{\frac{E}{\rho} \frac{t}{l^{2}}} \sqrt{1+0,295\left(\frac{l}{t}\right)^{2} \varepsilon}
$$

However, a remaining small discrepancy remains between the calculated and measured values. Consequently, doubly clamped beams cannot be used without restrictions for sensing of a mass loading by a homogeneous layer on the resonator.

However, the detection of single particles can be realized for a wide range of masses, from metal particles up to single cells. A line of Chinese hamster ovary (CHO-K1) cells in Dulbecco's modified Eagle's medium (DMEM) cells with 10 per cent fetal calf serum (FCS) has been selected for detailed testing. Biocompatibility tests have shown an excellent suitability of AlN and an adhesion without any adhesive agent $[\mathbf{2 1}]$.

Figure 7 shows AlN beams loaded by a different number of $\mathrm{CHO}$ cells. The resonators were exposed to the CHO-K1 in DMEM for one day. The cells preferably adhere at the Au surface of the beams and remain sticking to it after methanol rinsing and drying. For the beam with one single adherent cell, the frequency shifts from $420 \mathrm{kHz}$ to $61.7 \mathrm{kHz}$ before and after adhesion, respectively, corresponding to a mass loading of $1.5 \mathrm{ng}$, which is a suitable weight of such a cell. This variation corresponds to a differential mass sensitivity of about $-0.24 \mathrm{kHz} / \mathrm{pg}$. 
a)

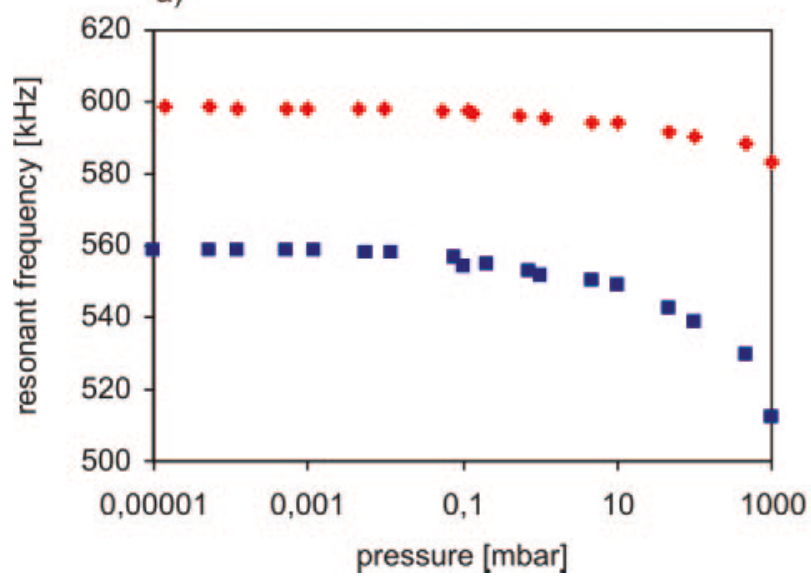

b)

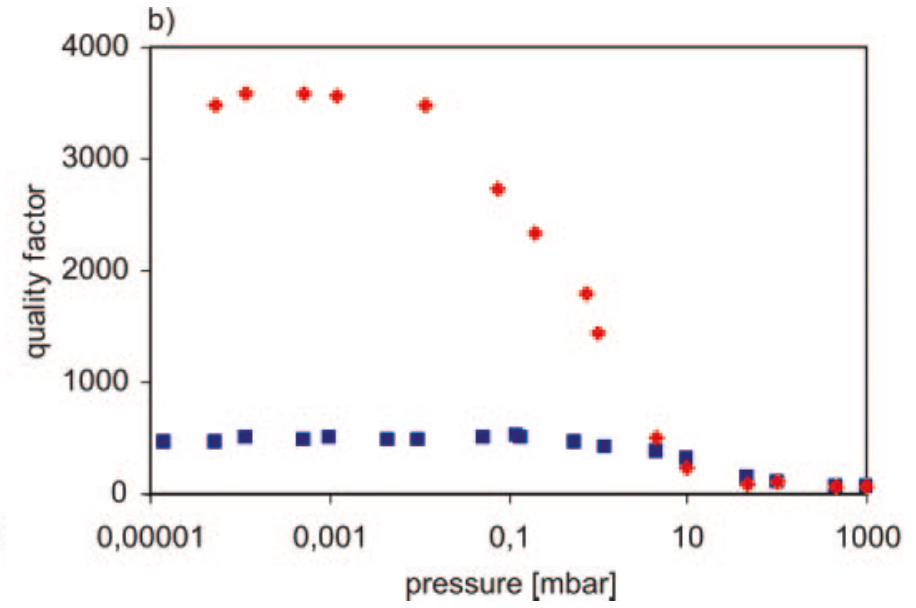

Fig. 8 Measured (a) quality factor and (b) resonant frequency of two AlN resonator beams with different strain in dependence of the pressure
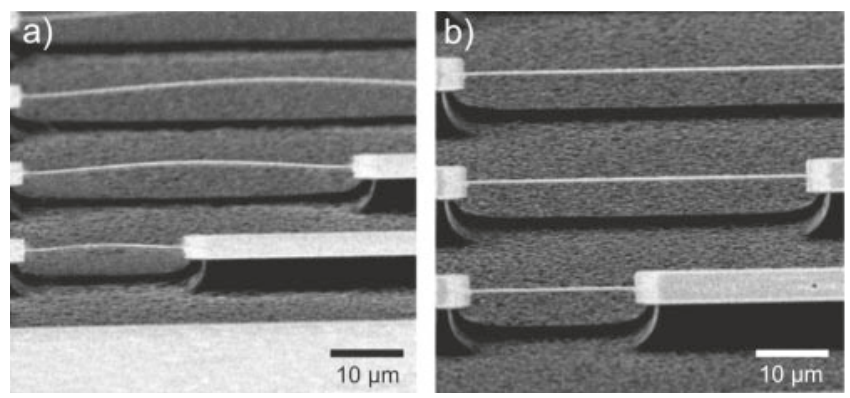

Fig. 9 (a) Compressive and (b) tensile strained AlN-beams corresponding to blue squares and red diamonds, respectively

\section{PRESSURE SENSING}

The resonant frequency and the quality factor of a cantilever oscillating in a gaseous environment are both dependent on the atmospheric pressure. Two AlN beams have been measured under identical pressure variations. Both samples had the same thicknesses of AlN and metallization, while the strain varied from compressive (blue squares) to tensile (red diamonds); see Fig. 8 and Fig. 9(a) and (b). According to equation (6), the compressive AlN exhibits lower resonant frequencies and quality factors.

To realize a highly sensitive pressure sensor, a strong response of the resonant parameters to pressure variations is required. Due to the reasonably strong dependence of $Q$ on $p$, a pressure sensor is proposed, which is based on the measurement of $Q$, in contrast to the commonly used frequency analysis.

The quality factor of an oscillating system depends on the density $\rho_{p}$ and the viscosity $\eta_{p}$ of the surrounding environment [22] according to

$$
Q=\frac{2}{3} \frac{\rho t w}{\frac{\eta_{p}}{f_{\text {res }}}+\frac{w}{2} \sqrt{\pi \rho_{p} \frac{\eta_{p}}{f_{\text {res }}}}}
$$

where $t, w$, and $\rho$ are the thickness, width, and density of the beam material, respectively. The linear dependence of the density to the pressure of the gaseous medium is described using

$$
\rho_{p}=\frac{p_{p}}{p_{0}} \cdot \rho_{0}
$$

where the index 0 refers to normal pressure. The density $\rho_{0}$ of air at normal pressure at $30^{\circ} \mathrm{C}$ is $1.164 \mathrm{~g} / \mathrm{cm}^{3}$. The viscosity of the surrounding medium $\eta_{p}$ is determined by

$$
\eta_{p}=\frac{N \cdot \bar{v}}{2} m_{a} \lambda
$$

with $m_{a}$ the atomic mass, which for air is ca. $4.81 \times 10^{-23} \mathrm{~g}, N$ the mean particle density, $\bar{v}$ the mean particle velocity, and $\lambda$ the mean free path. Theoretically, $N$ decreases, and $\lambda$ increases, for increasing pressure in the same way, and the viscosity remains constant. In a real system, the ratio of the size of the vacuum chamber $d_{c}$ to the mean free path has to be taken into account. Thus, the viscosity can be written as [23]

$$
\eta_{p}=\frac{N \cdot \bar{V}}{2} m_{a} \lambda \frac{d_{c}}{d_{c}+2 \lambda\left(\frac{2}{a_{p}}-1\right)}
$$

The variable $a_{p}$ accounts for the surface roughness of the chamber sidewalls, with values ranging from 1 (smooth) to 0 (rough). In this case, $a_{p}$ is supposed to assume values from 1 to 0.9 , which are the values for a smooth steel surface commonly used for the sidewalls in a vacuum chamber. The smallest dimension, which describes the distance of the beam to the substrate, is here represented by $d_{c}$ and takes values from 5 to $8 \mu \mathrm{m}$.

Passing through the different pressure ranges, the quality factor is specified by various parameters. At 

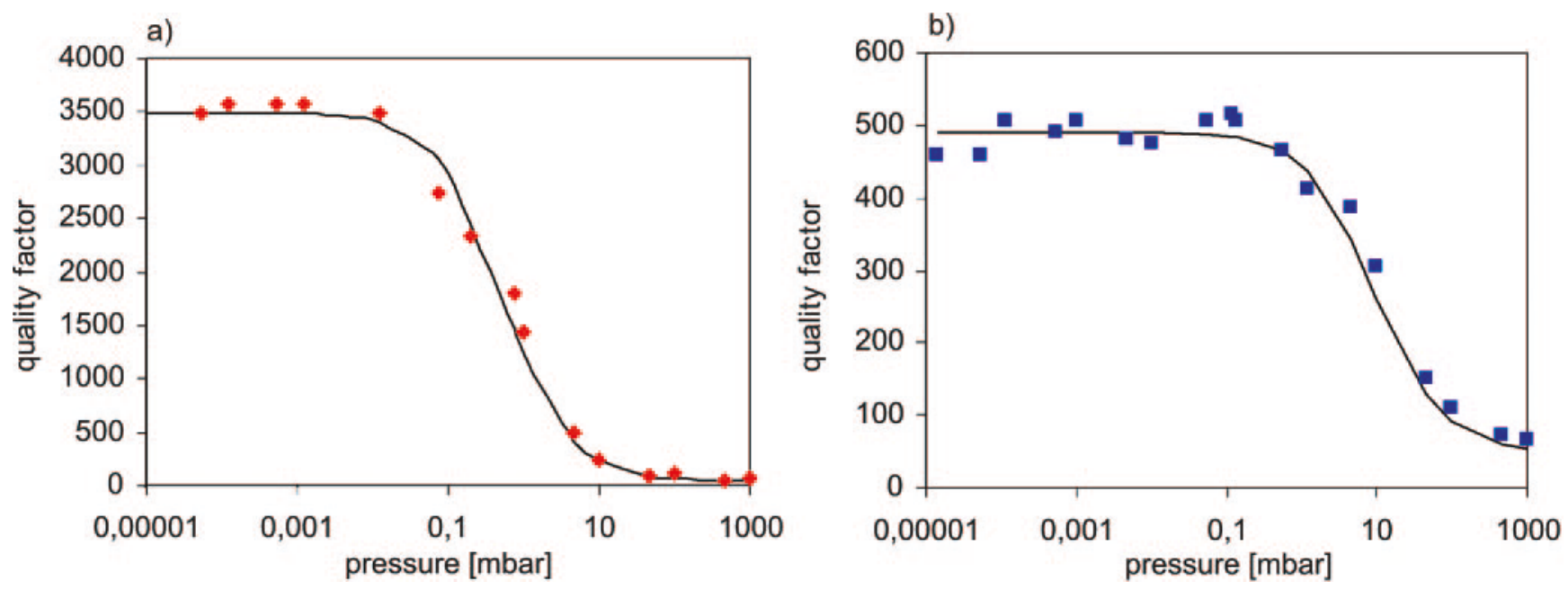

Fig. 10 Measured (symbols) and computed (curves) pressure dependence of the quality factors of (a) tensile and (b) compressive strained AlN resonators

normal pressure, the quality factor is limited by viscous damping. At very low pressures, the quality factor of the resonator reaches a constant limit due to the pressure-independent intrinsic losses. Considering both influences, the viscous damping $Q_{\text {visc }}$ as well as the intrinsic damping $Q_{\mathrm{bar}}$, the resulting quality factor $Q$ can be written as

$$
\frac{1}{Q}=\frac{1}{Q_{\text {visc }}}+\frac{1}{Q_{\text {bar }}}
$$

Figures 10(a) and 10(b) show measured quality factors of an AlN beam with a length and width of $250 \mu \mathrm{m}$ and $8 \mu \mathrm{m}$, respectively. The material system consisted of $225 \mathrm{~nm}$ AlN and a $50 \mathrm{~nm}$ conductive $\mathrm{Au}$ layer.

The solid lines represent simulated results using measured quality factors at the pressure range from $10^{-4} \mathrm{mbar}$ up to $10^{+3} \mathrm{mbar}$ with $a_{p}=1$ and $d=7 \mu \mathrm{m}$, which seems to correspond to the distance between the beam and the substrate as smallest distance in the system. It can be seen that the simulation provides excellent agreement to the measured quality factors for both the compressive and the tensile strained AlN. So we have realized a sensitive pressure sensor at the pressure range of 0.1 to 10 mbar, which shows the expected linear indirect proportional dependence of the quality factor on pressure with a sensitivity of 10 per cent/mbar.

\section{CONCLUSION}

Due to the significant influence of additional strain, doubly clamped beams do not seem to be a favourable design concerning homogeneous mass loadings.
These beams are, however, reliable and highly sensitive for mass loadings of single cells or metal particles. For further homogeneous mass loadings, other designs, which are able to relax, should be used to avoid deviation caused by additional stress. The high sensitivity of the quality factor of the beams on variations of the pressure could be used to perform a pressure sensor, which allows very differential measurements of the pressure in the pressure range from $10^{-2}$ to 10 mbar.

\section{ACKNOWLEDGEMENTS}

This work has been funded by the German Science Foundation (DFG), Priority Program 1157, 'Integrated electroceramic functional structures' (AM105/2). Additional support from the German Federal Ministry of Education and Research (grant no. 50YB0509) is greatly appreciated.

\section{REFERENCES}

1 Gardener, J. W. Microsensors: Principle and application, 1994, (John Wiley \& Sons, New York).

2 Lyshevski, S.E. Nano- and Micro-Electromechanical Systems: Fundamentals of Nano- and Microengineering, 2nd edition, Vol. 8, 2005, (CRC Press, Boca Raton, FL, USA).

3 Gupta, A. K., Nair, P. R., Akin, D., Ladisch, M. R., Broyles, S., Alam, M. A., and Bashir, R. Anomalous resonance in a nanomechanical biosensor. In Proceedings of the National Academy of Sciences, 2006, 103, 13363.

4 Ilic, B., Craighead, H. G., Krylov, S., Senaratne, W., Ober, C., and Neuzil, P. Attogram detection using nanoelectromechanical oscillators. J. Appl. Phys., 2004, 95, 3694 . 
5 Li, S., Orona, L., Li, Z., and Cheng, Z.-Y. Biosensor based on magnetostrictive microcantilever. Appl. Phys. Lett., 2006, 88, 073507.

6 Du, J., Ko, W. H., Mehregany, M., and Zorman, C. A. Poly-SiC capacitive pressure sensors made by wafer bonding. In Proceedings of the 4th IEEE Conference on sensors, Anaheim, CA, 31 October-3 November 2005, 1267-1270.

7 Young, D. J., Du, J., Zorman, C. A., and Ko, W. H. Hightemperature single crystal 3C-SiC capacitive pressure sensor. IEEE Sensors J., 2004, 4, 464-470.

8 Völklein, F. and Schnelle, W. A. Vacuum microsensor for the low vacuum range. Sens. Mater., 1991, 3, 41.

9 Wilfert, St. and Edelmann, Chr. Miniaturized vacuum gauges. J. Vac. Sci. Technol. A, 2004, 22, 309.

10 Adrega, T., Chu, V., and Conde, J. P. Electrostatically actuated resonance of amorphous silicon microresonators in water. Appl. Phys. Lett., 2006, 89, 143109.

11 Ekinci, K. L. and Roukes, M. L. Nanoelectromechanical systems. Rev. Scien. Instrum., 2005, 76, 061101.

12 Cleland, A.N., Pophristic, M., and Ferguson, I. Singlecrystal aluminum nitride nanomechanical resonators. Appl. Phys. Lett., 2001, 79, 2070-2072.

13 Förster, Ch., Cimalla, V., Ambacher, O., and Pezoldt, J. Low temperature chemical vapor deposition of 3C-SiC on Si substrates. Mater. Sci. Forum, 2005, 483-485, 201-204.

14 Brückner, K., Stephan, R., Hein, M. A., Förster, Ch., Cimalla, V., and Ambacher, O. Micro-electromechanical $\mathrm{RF}$ resonators from $\mathrm{SiC} / \mathrm{Si}$ heterostructures. In Proceedings of the 5th Workshop on MEMS for Millimeter WAVE communications, MEMSWAVE 2004, Uppsala, Sweden, 30 June-2 July 2004, H5-H8.

15 Förster, Ch., Cimalla, V., Brückner, K., Lebedev, V., Stephan, R., Hein, M., and Ambacher, O. Processing of novel SiC and group III-nitride based micro- and nanomechanical devices. Phys. Stat. Sol., 2005(a), 202, 671-676.

16 Zhu, K., Kuryatkov, V., Borisov, B., Kipshidze, G., Nikishin, S. A., Temkin, H., and Holtz, M. Plasma etching of AlN/AlGaInN superlattices for device fabrication. Appl. Phys. Lett., 2002, 81, 4688-4690.

17 Brückner, K., Förster, Ch., Tonisch, K., Stephan, R., Ambacher, O., and Hein, M. Electromechanical resonances of $\mathrm{SiC}$ and $\mathrm{AIN}$ beams under ambient conditions. In Proceedings of the 35th European Microwave Conference. Paris, 4-6 October 2005, 3, 1531-1534.

18 Ekinci, K. L., Yang, Y. T., Huang, X. M. H., and Roukes, M. L. Balanced electronic detection of displacement in nanoelectromechanical systems. Appl. Phys. Lett., 2002, 81, 2253-2255.

19 Brückner, K., Cimalla, V., Niebelschütz, F., Stephan, R., Tonisch, K., Ambacher, O., and Hein, M. Strain and pressure dependent RF response of microelectromechanical resonators for sensing applications. J. Micromech. Microeng., 2007. [In press]

20 Ikehara, T., Zwijze, R. A. F., and Ikeda, K. New methods for an accurate determination of residual strain in polycrystalline silicon films by analysing resonant frequencies of micromachined beams. J. Micromech. Microeng, 2001, 11, 55.

21 Cimalla, I., Will, F., Tonisch, K., Niebelschütz, M., Cimalla, V., Lebedev, V., Kittler, G., Himmerlich, M.,
Krischok, S., Schaefer, J. A., Gebinoga, M., Schober, A., Friedrich, Th., and Ambacher, 0. AlGaN/GaN biosensor - effect of device processing steps on the surface properties and biocompatibility. Sens. Actuat. B, 2006, 123 (2) 740-748.

22 Cimalla, V., Niebelschütz, F., Tonisch, K., Foerster, Ch., Brueckner, K., Cimalla, I., Friedrich, T., Pezoldt, J., Stephan, R., Hein, M., and Ambacher, O. Nanoelectromechanical devices for sensing applications. Sens. Actuat. B, 2007, 126, 24-34.

23 Wutz, M., Adam, H., Walcher, W., and Steckelmacker, W. (Trans.) Theory and practice of vacuum technology, 1989 (Friedrich Vieweg \& Son Verlag, Braunschweig), 30-33.

\section{APPENDIX}

\section{Notation}

$a_{p} \quad$ surface roughness

$b \quad$ frequency bandwidth at $-3 \mathrm{~dB}$

$d$ thickness of the additional layer

$d_{c} \quad$ size of the vacuum chamber

$d_{s} \quad$ thickness of the substrate

E Young's modulus

$f_{\text {res }} \quad$ resonant frequency

$l \quad$ beam length

$L \quad$ resulting beam length

$m \quad$ mass

$m_{a} \quad$ atomic mass

$N \quad$ mean particle density

$p$ pressure

$p_{0} \quad$ normal pressure

$Q \quad$ quality factor

$Q_{\text {bar }} \quad$ intrinsic damping

$Q_{\text {visc }} \quad$ viscous damping

$R \quad$ radius of curvature

$t \quad$ beam thickness

$T \quad$ period of oscillation

$U_{1}, U_{2}$ voltage amplitudes

$\overline{\bar{\nu}} \quad$ mean particle velocity

$w \quad$ beam width

\section{$\beta \quad$ angle}

$\Delta d \quad$ height difference

$\Delta m \quad$ mass load

$\Delta \sigma \quad$ additional stress

$\varepsilon \quad$ strain

$\eta_{\mathrm{p}} \quad$ velocity of surrounding medium

$\lambda$ mean free path

$\rho \quad$ density of beam material

$\rho_{0} \quad$ density of air at normal pressure

$\rho_{\mathrm{p}} \quad$ density of the surrounding medium

$\sigma \quad$ stress

$\tau_{1}, \tau_{2} \quad$ times 